Available online at www.sciencedirect.com

\title{
Perioperative tumor cell dissemination in patients with primary or metastatic colorectal cancer
}

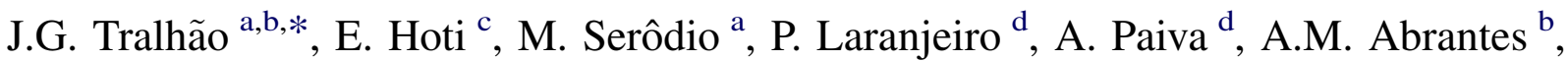 \\ M.L. Pais ${ }^{\mathrm{d}}$, M.F. Botelho ${ }^{\mathrm{b}}$, F. Castro Sousa ${ }^{\mathrm{a}}$ \\ ${ }^{a}$ Department of Surgery, Surgery 3, Faculty of Medicine, Coimbra University Hospital, Coimbra, Portugal \\ ${ }^{\mathrm{b}}$ Biophysics and Biomathematics Institute, IBILI, Faculty of Medicine, University of Coimbra, Coimbra, Portugal \\ ${ }^{\mathrm{c}}$ Liver Transplant Unit, Saint Vincent's University Hospital, Elm Park, Dublin 4, Ireland \\ ${ }^{\mathrm{d}}$ Center of Histocompatibility of Center, Coimbra, Portugal
}

Accepted 2 July 2009

\begin{abstract}
Introduction: Although there is general correlation between the TNM stage of colorectal cancer (CRC) and its prognosis, there is often significant variability of tumor behaviour and individual patient outcome, which is unaccounted for by pathologic factors alone. Our aim was to estimate perioperative tumor cell dissemination in patients with primary or CRC liver metastases as a possible factor influencing the outcome.

Methods: Forty patients were prospectively enrolled in the study from the year 2007 to 2008 . Eighteen patients had histologically proven CRC (50\% rectal, 44\% colonic, 6\% colonic and rectal). Sixteen patients (47\%) had CRC liver metastases only. The remaining six patients who underwent colon or liver resection for benign conditions, acted as the control group. All patients with malignant pathologies had R0 resections. Blood samples were taken before the surgical incision (T0), immediately after tumor resection (T1) and at the end of the surgical intervention (T2). Data acquisition was performed using a dual-laser FACSCalibur flow cytometer. Circulating malignant cells were identified as being CD45-/cytokeratin+.

Results: The analysis of patients overall (CRC resection subgroup and hepatectomy subgroup) revealed that there was no statistically significant difference of the tumoral cell count in the blood per million of hematopoietic cells at T0, T1 and T2.

Conclusions: This study demonstrates no differences in the detected circulating numbers of tumor cells at different stages of surgical intervention.

(C) 2009 Elsevier Ltd. All rights reserved.
\end{abstract}

Keywords: Colorectal cancer; Circulating tumor cells; Flow cytometry detection

\section{Introduction}

Colorectal cancer (CRC) is a common and often lifelimiting disease. Approximately $20-45 \%$ of patients with CRC undergoing curative resection subsequently develop local recurrence or metastatic disease in lymph nodes, liver, lung and peritoneum. ${ }^{1,2}$

Although there is general correlation between the TNM stage and prognosis, there is often significant variability of

\footnotetext{
* Corresponding author. Department of Surgery, Surgery 3, Hospitais da Universidade de Coimbra, Praceta Prof. Mota Pinto, 3000-075 Coimbra, Portugal. Tel.: +351 239400 417; fax: +351239480258.

E-mail address: jglrt@ hotmail.com (J.G. Tralhão).
}

tumor behaviour and individual patient outcome, which is unaccounted for by pathologic factors alone. The detection of lymph node (LN) metastases constitutes the most important prognostic factor in CRC and as the primary indicator of disease spread, LN status determines the choice of postoperative adjuvant chemotherapy. However, the limitations of TNM staging are emphasised by the considerable prognostic heterogeneity of patients within a given tumor stage (not all patients with LN-negative tumors are cured and not all patients with $\mathrm{LN}$-positive tumors die from their disease). This resulted in a number of efforts to develop more accurate staging protocols. ${ }^{3-6}$

Tumor progression after curative resection of CRC is caused by tumor cell dissemination, currently undetected 
by standard clinical staging techniques. The detection of disseminated tumor cells could help to identify a subgroup of patients at risk for disease relapse that could benefit from adjuvant therapy. ${ }^{6,7}$ In this context, flow cytometry is one of the methods used to identify subgroups at risk for disease relapse. Flow cytometry allows an accurate quantification of cells and at the same time a multi-parameter characterization of each cell present in the blood samples. This technique is widely used in the detection of rare events, such as minimal residual disease in hematological malignancies. Disseminated CRC cells have immunophenotypic characteristics distinct from those of hematopoietic cells, which allow their identification and quantification in peripheral blood by flow cytometry.

The aim of the study was to determine whether resection of the primary tumor or CRC liver metastases would lead to an increased dissemination of cancer cells.

\section{Patients and methods}

\section{Patients}

This prospective study included 40 patients who had undergone surgery in our institution from the year 2007 to 2008. There were 24 male patients with a median age and 16 female patients with a mean age of $64 \pm 10$ (range: 41-90).

Eighteen patients had histologically proven colorectal cancer $(50 \%$ rectal, $44 \%$ colonic, $6 \%$ colonic and rectal). Sixteen patients had colorectal liver metastases only. The remaining six patients, who acted as the control group had benign conditions (sigmoid diverticulosis, hepatic adenoma and hemangioma).

All patients were preoperatively staged by biological and radiological examinations. In addition, patients with rectal cancer received mandatory magnetic resonance imaging (MRI). Similarly, MRI was performed also for patients with non-conclusive computed tomography imaging (CT scan) concerning liver metastases originating from either rectal or colonic primary tumors.

Seven patients diagnosed with locally advanced rectal cancer (T3, T4, LN positive) received preoperative radiochemotherapy.

All patients operated for malignancy had R0 resections, performed according to international standards with at least $10 \mathrm{~cm}$ resection margins for colonic tumors or a distal resection margin of at least $2 \mathrm{~cm}$ associated with pericolic, perirectal and perivascular truncal lymphadenectomy for rectal cancers. High vascular ligation and no touch technique were used for all resections. Similarly, in the group of patients with liver metastases, liver resection was performed with at least $1 \mathrm{~cm}$ surgical margin.

Liver interventions included: right hepatectomy $(n=4)$, extended left hepatectomy $(n=1)$, left hepatectomy $(n=1)$, central hepatectomy $(n=1)$, left lobectomy $(n=4)$, bisegmentectomy $(n=3)$, segmentectomy $(n=2)$ and sub-segmentectomy $(n=4)$. Of the four patients who had left lobectomy, two had associated right hemicolectomy.

The colorectal procedures included: total coloproctectomy with ileostomy $(n=2)$, total colectomy $(n=2)$, subtotal colectomy $(n=1)$, right hemicolectomy $(n=1)$, left hemicolectomy $(n=3)$, anterior resection $(n=6)$, anterior resection with ileostomy $(n=1)$ and lastly abdominal perineal resection $(n=2)$.

\section{Blood sampling and processing}

Blood was sampled using central venous catheters before placing the surgical incision (T0), immediately after tumor resection (T1) and at the end of the surgical intervention (T2) and each time a volume of $12 \mathrm{ml}$ was obtained. As negative control, venous blood specimens were collected from the six patients who underwent surgery for benign conditions before (T0), during (T1) and after surgery (T2).

Peripheral blood samples were centrifuged at $540 \times \mathrm{g}$ for $10 \mathrm{~min}$, the buffy coat was collected and placed in a $50 \mathrm{ml}$ tube. Fixative-free $\mathrm{NH}_{4} \mathrm{Cl}$ lysing solution was added to each tube $(10 \mathrm{ml}$ of lysing solution per $1 \mathrm{ml}$ of buffy coat) and the samples were incubated for $45 \mathrm{~min}$ at room temperature (RT) to lyse the remaining red blood cells.

After the incubation period, the samples were centrifuged at $540 \times \mathrm{g}$ for $10 \mathrm{~min}$. The supernatant was discarded and the cell pellet washed with $10 \mathrm{ml}$ of phosphate-buffered saline (PBS, Gibco, Paisley, Scotland, UK) $(540 \times \mathrm{g}$ for $10 \mathrm{~min})$.

The cell pellet was stained with anti-CD45 (clone 30F11, BDB, San Jose, CA, USA) conjugated with peridinin chlorophyll protein cyanine 5.5 (PerCP Cy5.5) and anticytokeratin (clone MNF116, DakoCytomation, Glostrup, Denmark) conjugated with fluorescein isothiocyanate and incubated in the dark at RT for $30 \mathrm{~min}$. At last, the samples were washed twice in $10 \mathrm{ml}$ of PBS and the cell pellet resuspended in $1 \mathrm{ml}$ of PBS.

Data analysis was performed in a dual-laser FACSCalibur flow cytometer (BDB, San Jose, CA, USA) using the CellQuest software (BDB, San Jose, CA, USA). First, a total of 20000 events, corresponding to all nucleated cells in the sample, was stored. To increase the sensitivity of the technique, in a second step of acquisition, only cytokeratin + cells were acquired using an electronic live gate.

Data analysis was performed using the Paint-a-Gate Pro software (BDB, San Jose, CA, USA). Circulating CRC cells were identified as being CD45-/cytokeratin+. The monoclonal antibody anti-cytokeratin used recognized an epitope present in keratin 5, 6, 8 and 17.

\section{Statistical analysis}

Continuous data were presented as mean and standard deviation (SD). Skewed and non-gaussian continuous data 
were analyzed using non-parametric tests (Mann-Whitney U test or the Kruskal-Wallis test, whenever there were two, three or more samples to compare); Chi-square test was applied for the purpose of comparing proportions. Statistical analysis was performed using Statistica ${ }^{\circledR}$, version 7. A $p$ value of 0.05 was considered as statistically significant.

\section{Ethics}

Written informed consent was obtained for all the enrolled patients. The protocol of the study conformed to the ethical guidelines of the 1975 Declaration of Helsinki and also with the guidelines of our institution.

\section{Results}

\section{Patients of the control group}

In this group of patients $(n=6)$, none of the studied tumor markers were detected at any of the mentioned surgical times (T0, T1 and T2).

\section{Patients with colorectal cancer or CRC liver metastases}

We analyzed 102 blood samples from 34 patients with malignancy and detected circulating cancer cells in 31 blood samples from 34 patients who underwent curative surgical resection.

The median value of the tumoral cell count in the blood per million of hematopoietic cells was $7 \pm 7$ (range 0-26) at T0 (before the placement of surgical incision). At T1 (immediately after the removal of the specimen) the tumoral cell count in the blood per million of hematopoietic cells was $4 \pm 4$ (range 0-13) and lastly at T2 (end of the surgical intervention) the tumoral cell count in the blood per million of hematopoietic cells was $4 \pm 6$ (range $0-26)$. However, this value was statistically not significant $(p<0.501)$.

\section{Patients with colorectal cancer}

In this subgroup of patients the median value of the tumoral cell count in the blood per million of hematopoietic cells was $6 \pm 8$ (range $0-26$ ) at T0. Instead, at T1 the tumoral cell count in the blood per million of hematopoietic cells was $2 \pm 2$ (range $0-6$ ) and lastly at T2 the tumoral cell count in the blood per million of hematopoietic cells was $3 \pm 4$ (range $0-10$ ). Similar to the overall group of patients the value of tumoral cell count at T2 was statistically non-significant $(p<0.141)$.

\section{Patients with colorectal liver metastases}

The analysis in this subgroup of patients revealed that the median value of the tumoral cell count in the blood per million of hematopoietic cells was $9 \pm 4$ (range $0-14)$ at T0. Instead at T1 the tumoral cell count in the blood per million of hematopoietic cells was $7 \pm 4$ (range $0-13$ ) and lastly at T2 the tumoral cell count in the blood per million of hematopoietic cells was $6 \pm 9$ (range 0-26), which is statistically not significant $(p<0.088)$.

\section{Discussion}

In this study we have investigated the hypothesis that curative resection of a primary or secondary colorectal tumor can lead to shedding of malignant cells into the peripheral circulation. Our results obtained by using flow cytometry with the pancytokeratin antibody would suggest that such procedures are not associated or do not disseminate tumoral cells in the peripheral blood.

\section{Prognostic factors and management of colorectal cancer}

Tumor progression can result from disseminated tumor cells in lymph nodes, blood or bone marrow, sites which are not detected by current staging methods. The objective of adjuvant therapy is to eradicate viable disseminated tumor cells, thereby decreasing disease relapse and improving patients' survival. ${ }^{8}$ Candidates for post-operative adjuvant therapy are usually patients at high risk for disease relapse, as judged by current clinical and pathological staging. In the group of patients without distant metastases, lymph node metastases are the most important prognostic factor. ${ }^{9}$ Consequently, adjuvant chemotherapy is recommended for patients with positive lymph nodes. For patients with colon cancer stage I or II, adjuvant chemotherapy cannot achieve a survival benefit, and thus, adjuvant therapy is not recommended for these patients. Although considered at low risk, $10-20 \%$ of patients with colorectal cancer stage I and II ultimately will develop recurrent disease. ${ }^{10,11}$ It is in this population that prognostic markers may identify a subgroup of patients who are at a higher risk for disease relapse and who may also benefit from adjuvant therapy, especially from antitumoral agents with low systemic toxicity such as monoclonal antibodies, which have also proven to be effective against dormant tumor cells. ${ }^{6,7}$ In this regard, there are several studies that have demonstrated that tumor cell detection is clearly related to an early relapse and decreased survival of the respective patients. ${ }^{6,7,12,13}$ This new prognostic factor may change the surgical management of patients with colorectal liver metastases and may help to individualize the treatment of these patients with systemic or regional chemotherapy. ${ }^{14}$

\section{Flow cytometry in the detection of disseminated colorectal cancer cells}

Among the different approaches to screen disseminated colorectal cancer cells in bone marrow aspirates, peripheral 
and mesenteric venous blood, immunocytochemistry is the most widely used method. This method has the advantage to allow cell morphology characterization, but presents low sensitivity. ${ }^{6,15,16}$ RT-PCR based protocols have further improved the sensitivity and specificity of detection systems for disseminated cancer cells, allowing the identification of approximately one neoplastic cell in $10^{7}$ normal peripheral mononuclear blood cells. ${ }^{17}$

Several studies have proved that the sensitivity of flow cytometry is similar to PCR's, ranging from $10^{-4}$ to $10^{-5} \cdot{ }^{16,18,19,20}$ A study based on serial dilutions of breast cancer cells in normal peripheral blood, showed that, using the appropriated markers, flow cytometry presented a sensitivity ranging between $10^{-6}$ and $10^{-7} \cdot{ }^{21}$ Despite this, flow cytometry is not widely used in the detection of disseminated tumor cells in peripheral blood or bone marrow samples, probably because of the limited information in the literature regarding the phenotype of these cells. Because of the absence of tumor-specific target antigens, the disseminating tumor cells are identified based on the expression of epithelium-specific antigens such as cytoskeleton-associated cytokeratins, surface adhesion molecules, or growth factor receptors, whose quantitative expression obtained by flow cytometry is not well documented. Moreover, cytokeratin expression might vary along malignant transformations and different tumor stages. ${ }^{21,22}$ Other reasons that have limited the use of flow cytometry in this field are the absence of a consensus for the reagents and methods applied, the need for technical expertise and the long period required for sample acquisition, in order to obtain a large number of cells to achieve an acceptable sensitivity.

However, flow cytometry allows an accurate quantification of cells and enables the immunophenotypic characterization of each cell in the sample. Besides the improvement of flow cytometry technology, the development of new high-speed flow cytometers allowing the acquisition of 20000 events per second, has reduced dramatically the acquisition period. Altogether these factors make flow cytometry an attractive method for the quantification of rare events. This technique has become a method of choice for the detection of minimal residual disease in hematological malignant neoplasms ${ }^{17,19,23,24}$ and, more recently, an important tool in quantifying other rare events such as circulating endothelial cells, circulating progenitor cells, ${ }^{19,25}$ mesenchymal stem cells ${ }^{25}$ and disseminated tumor cells from CRC, ${ }^{26}$ small and non-small lung cancer, ${ }^{26}$ prostate cancer $^{27}$ and rhabdomyosarcoma. ${ }^{28}$

In this study, we used an anti-pancytokeratin monoclonal antibody able to recognize an epitope present in cytokeratin 5, 6, 8 and 17; and anti-CD45 monoclonal antibody. The analysis of the expression of both pancytokeratin and CD45 together with the light scatter properties enabled us to distinguish between non-hematopoietic cells (pancytokeratin+/CD45-) and peripheral blood cells (pancytokeratin-/CD45+). However, before we proceeded to the quantification of disseminated CRC cells in peripheral blood, we evaluated the pancytokeratin expression in tumor cells from tumor biopsies and verified the presence of two distinct populations: the first with a low pancytokeratin expression and the second with a higher expression. Based on previous proteomic studies in CCR showing a heightened cytokeratin 8 expression in tumor tissue compared to normal mucosa from the same individual, we assume that only those cells with higher pancytokeratin expression were CRC cells. ${ }^{29,30}$ Therefore, when peripheral blood samples were analyzed, we only considered as circulating CRC cells those with characteristic light scatter properties, CD45- and with high pancytokeratin expression.

In our results, none of the samples from patients with benign conditions who had surgical resection demonstrated the evaluated tumor markers. However, these tumor markers are not limited only to the gastrointestinal epithelium, as they have been found in a variety of cell types including urothelial, Merkel cells and leucocytes in $1982 .{ }^{31}$ On the other hand, several studies have also reported that in terms of molecular screening of circulating blood, the expression of several tumor markers is limited to patients with colorectal cancer with no expression seen in the controls. ${ }^{32,33}$

\section{Conclusion}

This prospective study using flow cytometry (a very specific and sensitive technique) to detect circulating tumor cells, demonstrated no differences in the circulating numbers of tumor cells detected at different times of the surgical intervention. These results would lead to the logical questions: what is the impact of the "no touch" technique in the oncological outcome of patients with primary colorectal cancer or liver metastases? Do we need to use perioperative adjuvant therapy or to change the surgical strategies to prevent intraoperative tumor cell shedding?

Our study does not suggest so; however, further studies should be performed to answer the raised questions.

\section{Conflict of interest}

Concerning the present manuscript, the authors declare that there are no personal, financial or non-financial competing interests or conflicts.

\section{References}

1. Feldman M, Friedman LS, Sleisenger MH, Feldman M, Scharschmidt BF. Sleisenger and Fordtran's Gastrointestinal and Liver Disease: Pathophysiology/Diagnosis/Management. 7th ed. Philadelphia: WB Saunders; 2002.

2. Goldberg RM, Fleming TR, Tangen CM, Moertel CG, Macdonald JS, Haller DG. Surgery for recurrent colon cancer: strategies for identifying resectable recurrence and success rates after resection. Eastern Cooperative Oncology Group, the North Central Cancer Treatment Group, and the Southwest Oncology Group. Ann Intern Med 1998; 129:27-35. 
3. Jen J, Kim H, Piantadosi S, Liu ZF, Levitt RC, Sistonen P. Allelic loss of chromosome $18 \mathrm{q}$ and prognosis in colorectal cancer. $N$ Engl J Med 1994;331:213-21.

4. Laurent-Puig P, Olschwang S, Delattre O, Remvikos Y, Asselain B, Melot T. Survival and acquired genetic alterations in colorectal cancer. Gastroenterology 1992;102:1136-41.

5. Gryfe R, Kim H, Hsieh ET, Aronson MD, Holowaty EJ, Bull SB. Tumor microsatellite instability and clinical outcome in young patients with colorectal cancer. $N$ Engl J Med 2000;342:69-77.

6. Yamaguchi K, Takagi Y, Aoki S, Futamura M, Saji S. Significant detection of circulating cancer cells in the blood by reverse transcriptasepolymerase chain reaction during colorectal cancer resection. Ann Surg 2000;232:58-65.

7. Weitz J, Koch M, Kienle P, Schrödel A, Willeke F, Benner A. Detection of hematogenic tumor cell dissemination in patients undergoing resection of liver metastases of colorectal cancer. Ann Surg 2000; 232:66-72.

8. Moertel CG. Chemotherapy for colorectal cancer. N Engl J Med 1994; 330: $1136-42$.

9. Ho SB, Hyslop A, Albrecht R, Jacobson A, Spencer M, Rothenberger DA. Quantification of colorectal cancer micrometastases in lymph nodes by nested and real-time reverse transcriptase-PCR analysis for carcinoembryonic antigen. Clin Cancer Res 2004;10:5777-84.

10. Kell MR, Winter DC, O'Sullivan GC, Shanahan F, Redmond HP. Biological behaviour and clinical implications of micrometastases. Br J Surg 2000;87:1629-39.

11. Maruthachalam K, Lash GE, Shenton BK, Horgan AF. Tumour cell dissemination following endoscopic stent insertion. Br J Surg 2007; 94:1151-4.

12. Guller U, Zajac P, Schnider A, Bösch B, Vorburger S, Zuber M. Disseminated single tumor cells as detected by real-time quantitative polymerase chain reaction represent a prognostic factor in patients undergoing surgery for colorectal cancer. Ann Surg 2002;236:768-75. discussion 775-766.

13. Iinuma H, Okinaga K, Egami H, Mimori K, Hayashi N, Nishida K. Usefulness and clinical significance of quantitative real-time RTPCR to detect isolated tumor cells in the peripheral blood and tumor drainage blood of patients with colorectal cancer. Int J Oncol 2006; 28:297-306.

14. Koch M, Kienle P, Hinz U, Antolovic D, Schmidt J, Herfarth C. Detection of hematogenous tumor cell dissemination predicts tumor relapse in patients undergoing surgical resection of colorectal liver metastases. Ann Surg 2005 Feb;241(2):206-7.

15. Riethdorf S, Wikman H, Pantel K. Review: biological relevance of disseminated tumor cells in cancer patients. Int J Cancer 2008;123: 1991-2006.

16. Al-Mawali A, Gillis D, Lewis I. The role of multiparameter flow cytometry for detection of minimal residual disease in acute myeloid leukemia. Am J Clin Pathol 2009;131:16-26.

17. Johnson PW, Burchill SA, Selby PJ. The molecular detection of circulating tumour cells. Br J Cancer 1995;72:268-76.

18. Kerst G, Kreyenberg H, Roth C, Well C, Dietz K, Coustan-Smith E. Concurrent detection of minimal residual disease (MRD) in childhood acute lymphoblastic leukaemia by flow cytometry and real-time PCR. Br J Haematol 2005;128:774-82.
19. Rawstron AC, Kennedy B, Evans PA, Davies FE, Richards SJ, Haynes AP. Quantitation of minimal disease levels in chronic lymphocytic leukemia using a sensitive flow cytometric assay improves the prediction of outcome and can be used to optimize therapy. Blood 2001;98:29-35.

20. Cruz I, Ciudad J, Cruz JJ, Ramos M, Gómez-Alonso A, Adansa JC. Evaluation of multiparameter flow cytometry for the detection of breast cancer tumor cells in blood samples. Am J Clin Pathol 2005; 123:66-74.

21. Stefansson IM, Salvesen HB, Akslen LA. Loss of p63 and cytokeratin $5 / 6$ expression is associated with more aggressive tumors in endometrial carcinoma patients. Int J Cancer 2006;118:1227-33.

22. Ciudad J, San Miguel JF, Lopez-Berges MC, Vidriales B, Valverde B, Ocqueteau M. Prognostic value of immunophenotypic detection of minimal residual disease in acute lymphoblastic leukemia. J Clin Oncol 1998;16:3774-81.

23. Maloum K, Charlotte F, Divine M, Cazin B, Lesty C, Merle-Beral H. A comparison of the sensitivity of flow cytometry and bone marrow biopsy in the detection of minimal residual disease in chronic lymphocytic leukemia. Haematologica 2006;91:860-1.

24. Jacques N, Vimond N, Conforti R, Griscelli F, Lecluse Y, Laplanche A. Quantification of circulating mature endothelial cells using a whole blood four-color flow cytometric assay. I Immunol Methods 2008;337:132-43.

25. Smiler D, Soltan M, Albitar M. Toward the identification of mesenchymal stem cells in bone marrow and peripheral blood for bone regeneration. Implant Dent 2008;17:236-47.

26. Dong Q, Huang J, Zhou Y, Li L, Bao G, Feng J. Hematogenous dissemination of lung cancer cells during surgery: quantitative detection by flow cytometry and prognostic significance. Lung Cancer 2002;37: 293-301.

27. Garcia JA, Rosenberg JE, Weinberg V, Scott J, Frohlich M, Park JW. Evaluation and significance of circulating epithelial cells in patients with hormone-refractory prostate cancer. BJU Int 2007;99:519-24.

28. Bozzi F, Collini P, Aiello A, Barzanò E, Gambirasio F, Podda M. Flow cytometric phenotype of rhabdomyosarcoma bone marrow metastatic cells and its implication in differential diagnosis with neuroblastoma. Anticancer Res 2008;28:1565-9.

29. Alfonso P, Núñez A, Madoz-Gurpide J, Lombardia L, Sánchez L, Casal I. Proteomic expression analysis of colorectal cancer by two-dimensional differential gel electrophoresis. Proteomics 2005;5:2602-11.

30. Friedman D, Hill S, Keller J, Merchant NB, Levy SE, Coffey RJ. Proteome analysis of human colon cancer by two-dimensional difference gel electrophoresis and mass spectrometry. Proteomics 2004;4: 793-811.

31. Moll R, Franke WW, Schiller DL, Geiger B, Krepler R. The catalog of human cytokeratins: patterns of expression in normal epithelia, tumors and cultured cells. Cell 1982;31:11-24.

32. Moll R, Zimbelmann R, Goldschmidt MD, Keith M, Laufer J, Kasper M. The human gene encoding cytokeratin 20 and its expression during fetal development and in gastrointestinal carcinomas. Differentiation 1993;53:75-93.

33. Weitz J, Kienle P, Magener A, Koch M, Schrödel A, Willeke F. Detection of disseminated colorectal cancer cells in lymph nodes, blood and bone marrow. Clin Cancer Res 1999;5:1830-6. 